\title{
Construction of a Liquid-State NMR DNP Shuttle Spectrometer: First Experimental Results and Evaluation of Optimal Performance Characteristics
}

\author{
M. Reese ${ }^{1}$, D. Lennartz ${ }^{1,2}$, T. Marquardsen ${ }^{3}$, P. Höfer ${ }^{3}$, A. Tavernier $^{3}$, \\ P. $\mathrm{Carl}^{3}$, T. Schippmann ${ }^{3}$, M. Bennati ${ }^{1}$, T. Carlomagno ${ }^{1}$, F. Engelke ${ }^{3}$, and \\ C. Griesinger ${ }^{1}$ \\ ${ }^{1}$ Max Planck Institute for Biophysical Chemistry, Göttingen, Germany \\ ${ }^{2}$ Parexel International $\mathrm{GmbH}$, Berlin, Germany \\ ${ }^{3}$ Bruker, Rheinstetten, Germany
}

Received 4 November 2007; revised 21 March 2008

(C) Springer-Verlag 2008

\begin{abstract}
First results from a liquid-state shuttle dynamic nuclear polarization (DNP) spectrometer are presented. The device polarizes a water sample at $9.7 \mathrm{GHz}$ and $0.34 \mathrm{~T}$ in a commercial Bruker electron paramagnetic resonance (EPR) spectrometer and then transfers the sample via a homebuilt pneumatic shuttle device into the $600 \mathrm{MHz}$ and $14.09 \mathrm{~T}$ nuclear magnetic resonance (NMR) spectrometer with a conventional NMR probe for detection. The shuttle transfer time is approximately 115 ms. Initial experiments measure the postshuttle proton magnetization compared with the Boltzmann magnetization at $14.09 \mathrm{~T}$. The DNP enhancement factor at $0.34 \mathrm{~T}$ is reported for the nitroxide polarizer TEMPOL in water solution. Reduction of the magnetization during shuttling because of relaxation is quantified. Optimization of this apparatus is expected to bring the NMR enhancement factor for protons close to the theoretical enhancement maximum of -7.92 .
\end{abstract}

\section{Introduction}

Dynamic nuclear polarization (DNP) can be used to increase the sensitivity of nuclear magnetic resonance (NMR) detection. Recent successes of the DNP approach have been achieved in solid-state NMR as well as for a combined solidliquid-state experiment, where a frozen sample is polarized through the transfer mechanisms active in solid-state and subsequently melted to measure the final NMR spectrum in liquid-state. This last strategy, however, is applicable only to molecules that are compatible with freezing and melting processes, as, for example, many small molecules. As compared to the room-temperature Boltzmann magnetization effective enhancement factors of many hundreds have been reported for ${ }^{13} \mathrm{C}$ and ${ }^{15} \mathrm{~N}$ nuclei [1-3]. 
For biological molecules in solution, the freeze-thaw approach is not practicable due to the risk of the sample denaturation. Therefore, it is desirable to be able to transfer polarization from the electrons to the biomolecule's protons directly in solution. The transfer mechanism active in solution is the Overhauser effect, the utilization of which normally involves the saturation of the electron paramagnetic resonance (EPR) signals of a polarizing agent.

When the DNP transfer is performed at the same temperature as the detection of the NMR lines, the enhancement of the nuclear polarization is determined by gyromagnetic ratios of the electron and the nucleus, which is -658 for a proton nucleus and -2616 for a carbon nucleus. Complete saturation of the EPR line in aqueous solution is challenging due to the very efficient absorption of microwaves (MW) by water and the resulting strong heating. To alleviate this problem, optimal microwave cavities can be constructed to minimize the electric field, which is responsible for heating, and to optimize the magnetic field at the location of the sample. This design requires smaller sample volumes for higher microwave frequencies. However, small sample volumes also result in a considerable loss of sensitivity for NMR detection.

To compromise on the two different effects, we chose to perform DNP with pumping at the relatively low X-band frequency at $0.34 \mathrm{~T}$ and subsequent shuttling the sample back to $14.09 \mathrm{~T}$ for NMR detection. Furthermore, X-band offers the most evolved EPR instrumentation to test all possible conditions for optimizing the DNP performance at a first stage. The NMR sample shuttling has been pioneered for relaxation measurements by Purcell and Pound [4] (see also ref. 5 and references therein, as well as refs. 6 and 7) in 1951 and for chemically induced DNP by Vieth et al. [8].

DNP in liquids has been studied since the 1960s [9]. The maximum enhancement factor $\varepsilon$ achievable with continuous-wave microwave irradiation is given by [9]

$$
\varepsilon=\frac{I_{z}}{I_{0}}=1+\xi f_{S} \frac{\gamma_{s}}{\gamma_{I}}=1-\xi f_{S} \frac{\left|\gamma_{s}\right|}{\gamma_{I}}
$$

where $I_{z}$ and $I_{0}$ are the nuclear magnetization and the Boltzmann nuclear magnetization, respectively. Here $\gamma_{s}$ and $\gamma_{I}$ are the gyromagnetic ratios of the electron and the proton [10], respectively, $f$ is the leakage, $s$ is the saturation and $\xi$ is the coupling factor. The coupling factor $\xi$ depends on the involved relaxation mechanisms and can achieve values in the range of -1 (pure scalar interaction) up to +0.5 (pure dipolar interaction) depending on the effective correlation times. The experimentally available enhancement factors for TEMPOL have been studied recently [11, 12]. As reported in ref. 12, an enhancement factor for water of -100 or -140 was achieved at a pumping field of $0.34 \mathrm{~T}$ using 5 or $25 \mathrm{mM}$ ${ }^{15} \mathrm{~N}-16 \mathrm{D}-\mathrm{TEMPONE}$, respectively. With the DNP setup of the shuttle spectrometer, as described in Sect. 2, we could measure low-field enhancement factors of up to -60 for $5 \mathrm{mM}$ TEMPOL solution in $80 \% \mathrm{H}_{2} \mathrm{O}$ and $20 \% \mathrm{D}_{2} \mathrm{O}$ and of up to -130 for $25 \mathrm{mM}{ }^{15} \mathrm{~N}-16 \mathrm{D}$-TEMPONE solution in $100 \% \mathrm{H}_{2} \mathrm{O}$. 
Given the maximal proton magnetization $I_{z, \max }$ for $s=1, f=1$ and $\xi=-0.5$ (pure dipolar coupling) and using the electron and the proton Boltzmann magnetizations $I_{0}^{\mathrm{EPR}}$ and $I_{0}^{\mathrm{NMR}}$ at 0.34 and $14.09 \mathrm{~T}$, respectively, the maximum possible high-field enhancement factor $\varepsilon_{\max }^{\mathrm{NMR}}=I_{z, \max } / I_{0}^{\mathrm{NMR}}$ is proportional to the lowfield enhancement factor $\varepsilon_{\max }^{\mathrm{EPR}}$ and $I_{0}^{\mathrm{EPR}}$, because the maximum possible magnetization $I_{z \text {,max }}$ equals to $I_{0}^{\mathrm{EPR}} \varepsilon_{\max }^{\mathrm{EPR}}$. In the considered regime the Boltzmann magnetizations are proportional to the field strength. Magnetization loss due to relaxation during the sample transfer is neglected here. The maximum magnetization at low field, just after microwave irradiation and at the beginning of the sample transfer, is

$$
\begin{aligned}
I_{z, \max } & =\varepsilon_{\max }^{\mathrm{EPR}} I_{0}^{\mathrm{EPR}} \\
& =\left(1-0.5\left|\gamma_{s}\right| / \gamma_{I}\right) I_{0}^{\mathrm{EPR}} \\
& =\left(1-0.5\left|\gamma_{s}\right| / \gamma_{I}\right)\left(B^{\mathrm{EPR}} / B^{\mathrm{NMR}}\right) I_{0}^{\mathrm{NMR}} \\
& =\varepsilon_{\max }^{\mathrm{NMR}} I_{0}^{\mathrm{NMR}} .
\end{aligned}
$$

Hence the maximum high-field enhancement factor is $\varepsilon_{\max }^{\mathrm{NMR}}=\left(1-0.5\left|\gamma_{s}\right| / \gamma_{I}\right) \times$ $\left(B^{\mathrm{EPR}} / B^{\mathrm{NMR}}\right)=\varepsilon_{\max }^{\mathrm{EPR}}\left(B^{\mathrm{EPR}} / B^{\mathrm{NMR}}\right)$. Using the value of the maximum low-field enhancement factor $\varepsilon_{\max }^{\mathrm{EPR}}$ of -328.11 and chosen values of magnetic fields, the maximum high-field enhancement factor $\varepsilon_{\max }^{\mathrm{NMR}}$ becomes -7.92 . Both enhancement factors can never be achieved as, in practice, the low-field enhancement is limited by $s, f$ and $\xi$ and as the nuclear magnetization is relaxing to the equilibrium during the sample shuttling from the low-field to the high-field magnet. For the quantification of such relaxation losses we used the variable-field $T_{1}$ relaxation measurements of Luchinat and Parigi [13].

\section{Experimental}

We have implemented an X-band (9.6 GHz EPR, $114.7 \mathrm{MHz}{ }^{1} \mathrm{H}$ NMR) $600 \mathrm{MHz}$ ${ }^{1} \mathrm{H}$ NMR DNP shuttle spectrometer composed of a commercial EPR spectrometer, utilizing an electron-nuclear double-resonance (ENDOR) probehead optimized for ${ }^{1} \mathrm{H}$ detection, mounted on a platform above the cryostat of a $600 \mathrm{MHz} \mathrm{NMR}$ magnet. A pneumatic shuttle device connects the two spectrometers. A schematic drawing of the shuttle spectrometer is given in Fig. 1. A photograph of the setup in the laboratory is given in Fig. 2.

The shuttle DNP NMR spectrometer consists of three subsystems: the DNP spectrometer, the shuttle system and the NMR detection system.

The DNP spectrometer consists of a separate permanent magnet (with $B_{0}=$ $0.34 \mathrm{~T}$, horizontal direction), sweep coils to vary this field within a narrow range, an ENDOR cavity (including a dielectric resonator operating in $\mathrm{TE}_{011}$ mode at 9.6 GHz, X-band) serving to provide the microwave field and to pick up the ${ }^{1} \mathrm{H}$ NMR signal at $14.7 \mathrm{MHz}$ for reference purpose, a microwave guide connected to 


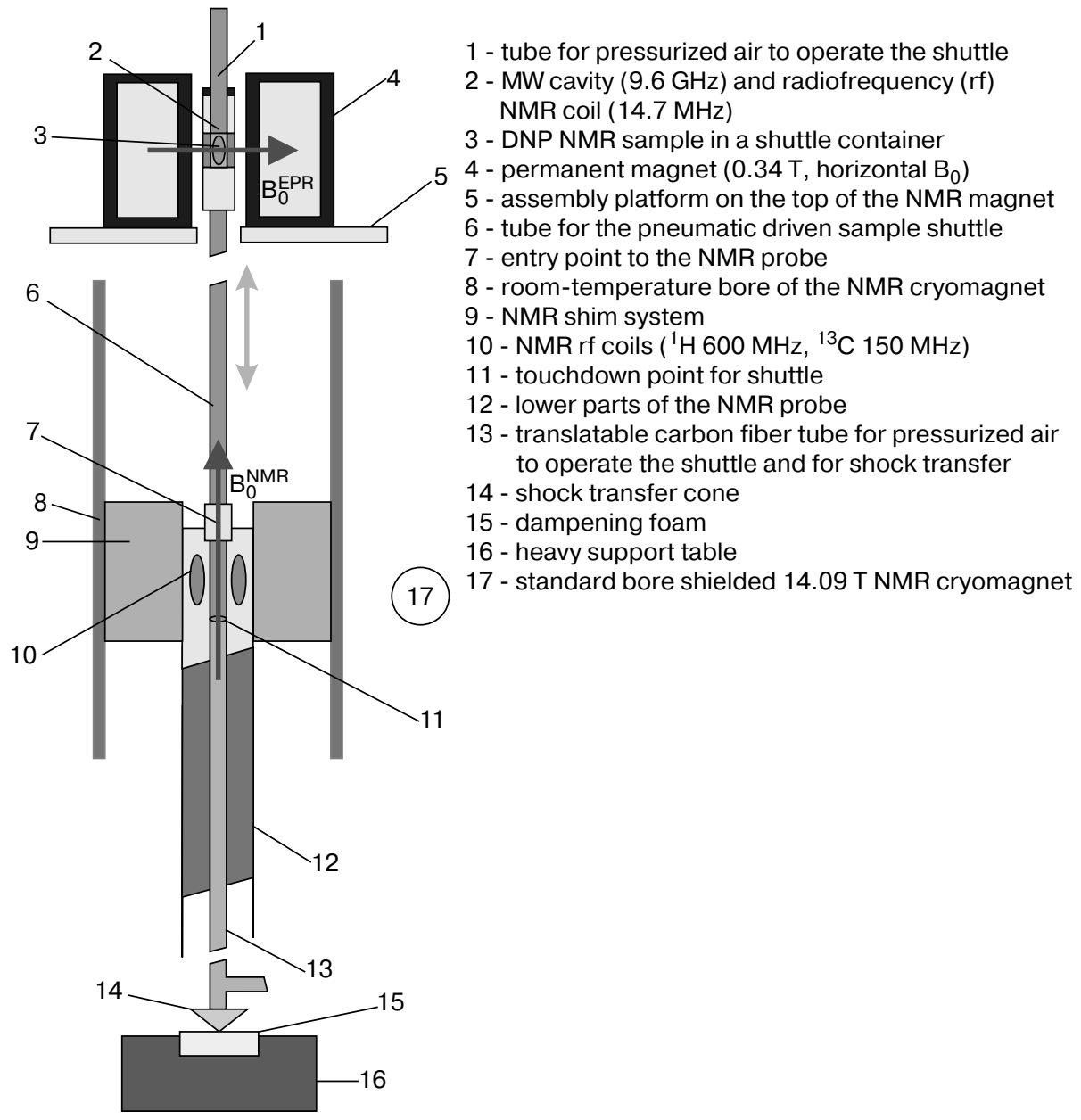

Fig. 1. Schematic drawing of the shuttle system connecting both magnetic centers and its environment.

an EPR bridge and a Bruker E-scan console to operate the MW equipment, as well as a Bruker Minispec for the low-field NMR part. The DNP system is mounted with the center of the EPR cavity $1.525 \mathrm{~m}$ above the center of a regular high-field NMR magnet.

The shuttle system consists of a shuttle container housing the DNP NMR sample, a shuttle tube with an inner diameter (i.d.) of $4.5 \mathrm{~mm}$ and integrated photosensors connecting the MW cavity with the NMR probe inside the NMR cryomagnet and a microprocessor controller with proportional pressure valves to control the sample shuttling.

The shuttle container with an outer diameter (o.d.) of $4.1 \mathrm{~mm}$ and i.d. of $2.4 \mathrm{~mm}$ is made of a quartz glass tubing with $4.2 \mathrm{~mm}$ o.d. Kel-F plugs on both sides. The container has an active volume of $60 \mu \mathrm{l}$ for low-dielectric-loss samples 


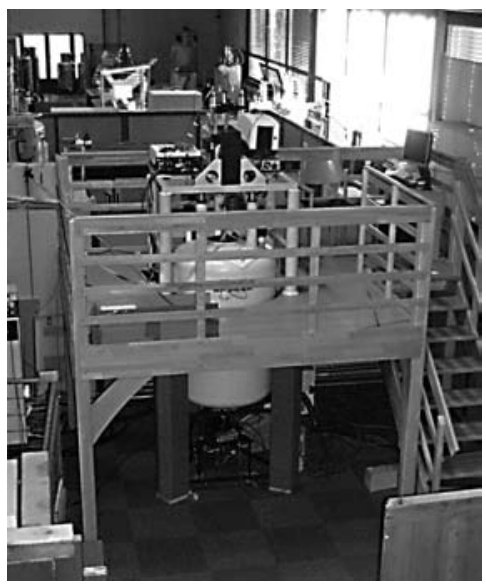

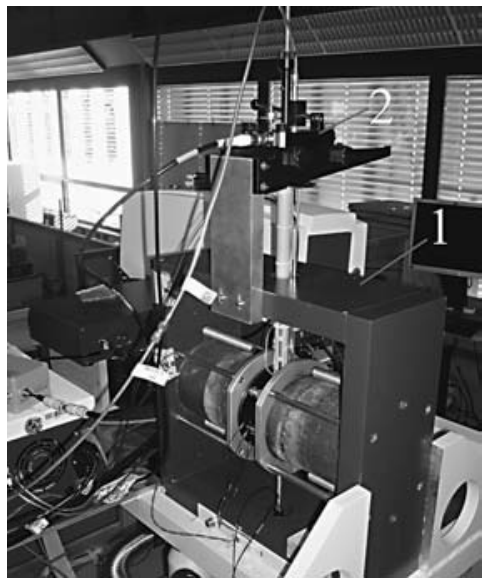

Fig. 2. DNP NMR spectrometer hardware setup in the laboratory. a View to the complete setup of the stacked magnets. b Top view to the EPR setup with the permanent magnet (1) and ENDOR probehead (2). In the back there are the EPR amplifier and console, as well as the NMR Minispec console. Personal computer seen on the very right-hand side is used for controlling both consoles.

(e.g., benzene). For high-loss samples (e.g., water) it is necessary to reduce the sample diameter because of the high losses arising in the EPR resonator. For this reason a second tube with an i.d. of $0.9 \mathrm{~mm}$ and an active volume of $10 \mu \mathrm{l}$ is inserted into the shuttle container. The shuttle container can translate and rotate freely in the shuttle tube.

The microprocessor shuttle controller directs the motion of the shuttle container by controlling two proportional pressure valves for the upward and downward motion. The shuttle controller is triggered by the NMR console. Accelerat-

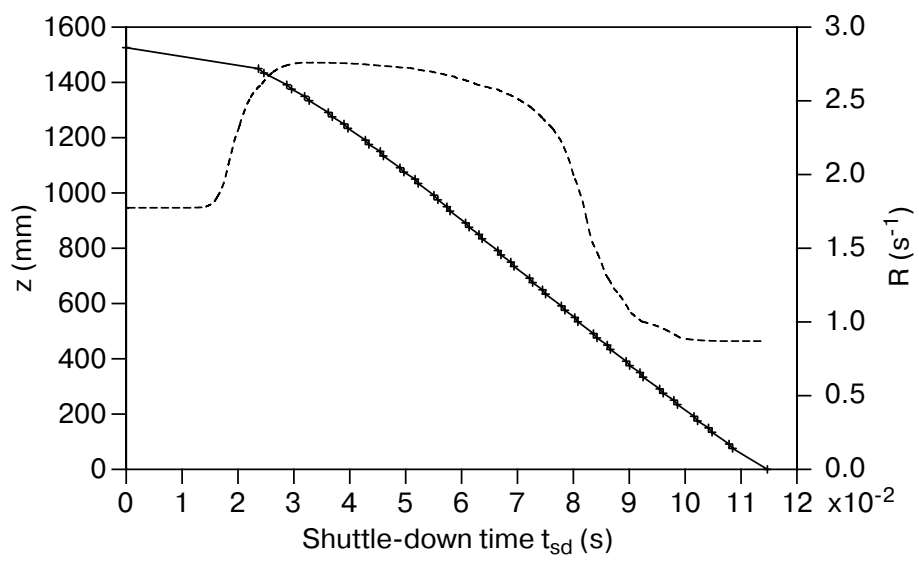

Fig. 3. Plot of the position of the sample $z$ (solid line) and the $T_{1}$ relaxation rate $R$ (dotted line) [13] during the transfer from the low to the high field. The position has been measured using sixteen photosensors as described in the text. The transfer time is referenced to zero when the sample is no longer detected at the starting position. 
a

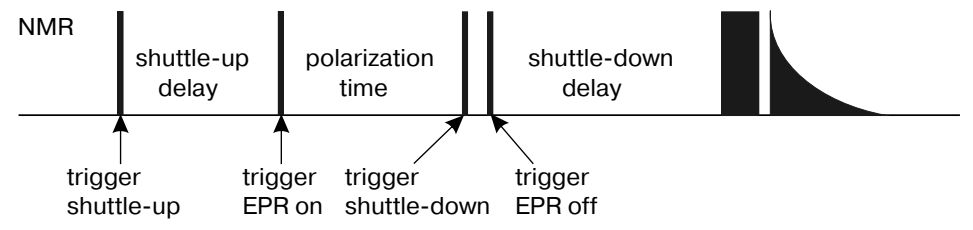

trigger

shuttle

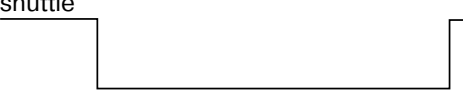

sample

position

up

down

\section{trigger}

EPR

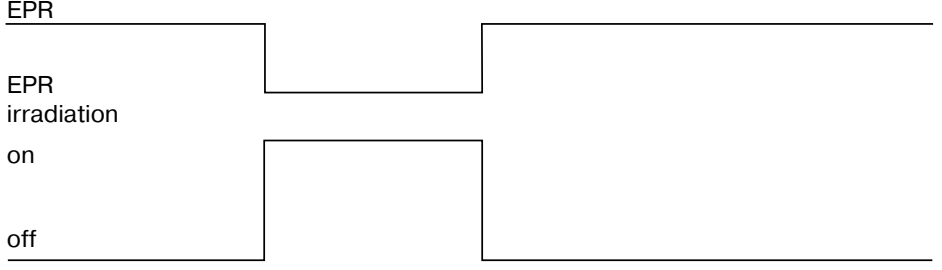

b

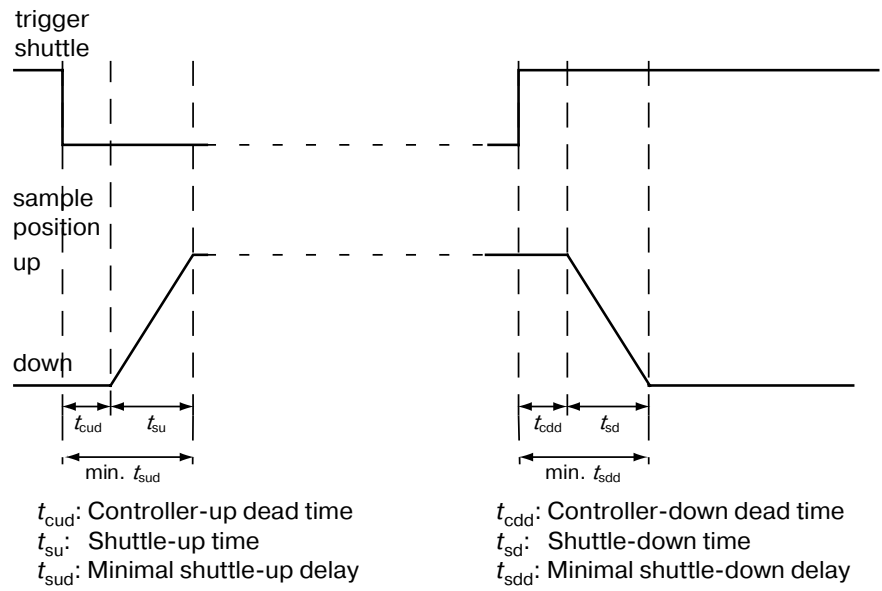

Fig. 4. a Timing diagram of the shuttle DNP experiment. The sample is shuttled up into the EPR spectrometer, and DNP enhancement is obtained by MW irradiation at $9.7 \mathrm{GHz}$ for 2 s. To avoid relaxational losses at $0.34 \mathrm{~T}$ the $\mathrm{MW}$ is switched off $50 \mathrm{~ms}$ after the sample has been triggered to leave the EPR magnet and is shuttling down to the NMR spectrometer. Subsequent to a variable delay after the sample arrival a $90^{\circ}\left({ }^{1} \mathrm{H}\right)$ pulse is applied and the NMR signal is detected. b Timing diagram of the shuttle motion. 
ing and decelerating of the shuttle container is determined by a feedback-less time step program.

Furthermore, the controller utilizes sixteen photosensors positioned along the whole shuttle tube length to measure and record the motion of the sample container (Fig. 3). The photosensors are triggered when the caps of the otherwise transparent sample tube interrupt the light beam. Entering and leaving of either cap gives each one the time-position coordinate. This gives four independent time-position measurements for a single sample passage for each sensor on the sample track. The sensors at the ending and starting position can only be entered and not be passed. Each of them only delivers a single time-position coordinate.

The NMR detection system consists of a $14.09 \mathrm{~T}$ NMR shielded cryomagnet $\left({ }^{1} \mathrm{H} 600 \mathrm{MHz}\right.$ "UltraShield", Bruker), a shuttle high-resolution NMR probe (selective inverse ${ }^{1} \mathrm{H} /{ }^{13} \mathrm{C}$ plus ${ }^{2} \mathrm{H}$ lock channel), and a standard $600 \mathrm{MHz}$ Bruker Avance console.

In order to ensure that the two-magnet setup (DNP permanent magnet on the top of the NMR cryomagnet) is a feasible hardware solution, the interaction between the two magnets has been considered in detail. Stray field measurements and simulations were performed to determine the torque and forces between the two magnets, before assembling the permanent magnet on the top of the NMR magnet. For the $1.525 \mathrm{~m}$ distance between the two magnetic centers, the torque and forces present no risk of damaging the cryomagnet. $B_{0}$ homogeneity at the magnetic center of the NMR cryomagnet does not pose a problem. Small inhomogeneities resulting from the stray field of the permanent magnet can be easily compensated by using the standard NMR shim system. We expect to achieve the spectral resolution of a conventional $600 \mathrm{MHz}$ NMR spectrometer, as we experienced in a non-DNP version of the shuttle setup.

The shuttle spectrometer is under the control of two consoles as well as the controller for the shuttle. Trigger signals for shuttling up and down as well as for switching on the microwave power are derived from the NMR spectrometer that is the master controller for the experiment. Shuttle times are in the order of 100 to $200 \mathrm{~ms}$.

We performed the shuttle DNP experiment in the following way. The sample was shuttled to the low-field position, irradiated with MW for $2 \mathrm{~s}$ and then shuttled to the detection field (Fig. 4a). There, after a variable relaxation recovery delay, which varied between $150 \mathrm{~ms}$ and $6.5 \mathrm{~s}$ including the shuttle-down delay $t_{\text {ssd }}$ (Fig. $4 \mathrm{~b}$ ), the signal was recorded with a $90^{\circ}$ pulse on the proton magnetization. A reference experiment without electron irradiation was also performed.

The timing diagram (Fig. 4b) indicates the time delays of the shuttle motion. The dead times of the shuttle controller are specified as the time periods from the up or down trigger signal to the first detected motion of the shuttle container. The controller-up dead time $\left(t_{\text {cud }}\right)$ and controller-down dead time $\left(t_{\text {cdd }}\right)$ are of the order of $50 \mathrm{~ms}$ for the upward motion and 20-30 ms for the downward motion. They are caused by a delay for complete opening of the valves after triggering, pressure buildup in the shuttle tube, and moving the shuttle container out of the detection range of light barriers at the end positions. As the shuttle-down pressure was chosen higher than the shuttle-up pressure the dead times vary. The dead 
times have no influence on the achieved shuttle DNP enhancements. During the shuttle-down dead time the MW irradiation is continued to avoid rapid relaxational losses at 0.34 T. MW irradiation is switched off only after the sample has actually left the EPR magnet. Thus the shuttle-down dead time does not reduce the efficiency of low-field DNP. The shuttle times are the durations during which the sample container is not detected at the end positions. These durations are the shuttleup time $\left(t_{\mathrm{su}}\right)$ and the shuttle-down time $\left(t_{\mathrm{sd}}\right)$ and depend on the pressure control. For the up-motion it amounts to ca. $500 \mathrm{~ms}$, and for the quickest shuttle downmotion to $115 \mathrm{~ms}$. The shuttle-up delay $\left(t_{\text {sud }}\right)$ is equal to the sum of $t_{\text {cud }}$ and $t_{\text {su. }}$. The timing was chosen so that between the sample arrival at the top position and MW irradiation a waiting time of about $2000 \mathrm{~ms}$ was achieved. The minimum delay for shuttle-down $\left(t_{\text {sdd }}\right)$ equals the sum of $t_{\text {cdd }}$ and $t_{\text {sd }}$.

To evaluate the effect of relaxation during shuttling, the trajectory of the sample from the EPR magnet to the center of the NMR magnet was measured with the help of sixteen photosensors. A typical time trace is shown in Fig. 3. The shuttling was done with a pressure of up to $5 \cdot 10^{5} \mathrm{~Pa}$ and lasted $115 \mathrm{~ms}$. The field-dependent $T_{1}$ values for water in a $5 \mathrm{mM}$ TEMPOL solution [13] were used to calculate the relaxation losses during the transfer of the sample from the EPR to the NMR magnet.

\section{Results and Discussion}

Enhancement was measured for a $5 \mathrm{mM}{ }^{14} \mathrm{~N}$-TEMPOL solution $\left(80 \% \mathrm{H}_{2} \mathrm{O}, 20 \%\right.$ $\mathrm{D}_{2} \mathrm{O}$ ) in the EPR magnet as well as after the shuttling at $600 \mathrm{MHz}$. We obtained an enhancement factor of -27 in the EPR magnet after $2000 \mathrm{~ms}$ of irradiation at a frequency of $9.7 \mathrm{GHz}$. We then conducted the experiment explained in Fig. 4a. For reference we also performed the experiment in the absence of MW irradiation. The time between the shuttle-down trigger and the application of the $90^{\circ}\left({ }^{1} \mathrm{H}\right)$ pulse was varied from $150 \mathrm{~ms}$ to $6.5 \mathrm{~s}$ to measure the magnetization recovery curves at $14.09 \mathrm{~T}$. The signal intensity with and without microwave irradiation was recorded. The integrals are plotted in Fig. 5.

Independently of DNP, the magnetization $I^{\mathrm{NMR}}(t)$ at $14.09 \mathrm{~T}$ recovers back to the Boltzmann equilibrium magnetization with a rate of approximately $0.87 \mathrm{~s}^{-1}$. This relaxation rate for water protons in a $5 \mathrm{mM}$ TEMPOL solution was determined by Luchinat and Parigi [13]. Using a rate of $0.875 \mathrm{~s}^{-1}$ a monoexponential fitting for the non-DNP relaxation measurement has been achieved. The starting magnetizations in the NMR magnet are different for the experiments with and without microwave irradiation. In the experiment without irradiation, the magnetization starts at approximately zero at $t=150 \mathrm{~ms}$ and converges to the Boltzmann equilibrium. With microwave irradiation, the magnetization has a negative sign, indicating a DNP effect. In this case the extrapolated magnetization at $t=139 \mathrm{~ms}$, just after the sample arrival, leads to a postshuttle enhancement of $\varepsilon^{\mathrm{NMR}}(t=139 \mathrm{~ms})=-0.41$ (Fig. 5). Although DNP could be detected using a short stabilization period of $61 \mathrm{~ms}$ after the sample arrival, a reliable measurement required a longer stabilization period of $111 \mathrm{~ms}$ at high field. Due to this delay the enhancement factor was reduced to the measured $\varepsilon^{\mathrm{NMR}}(t=250 \mathrm{~ms})=-0.38$. 


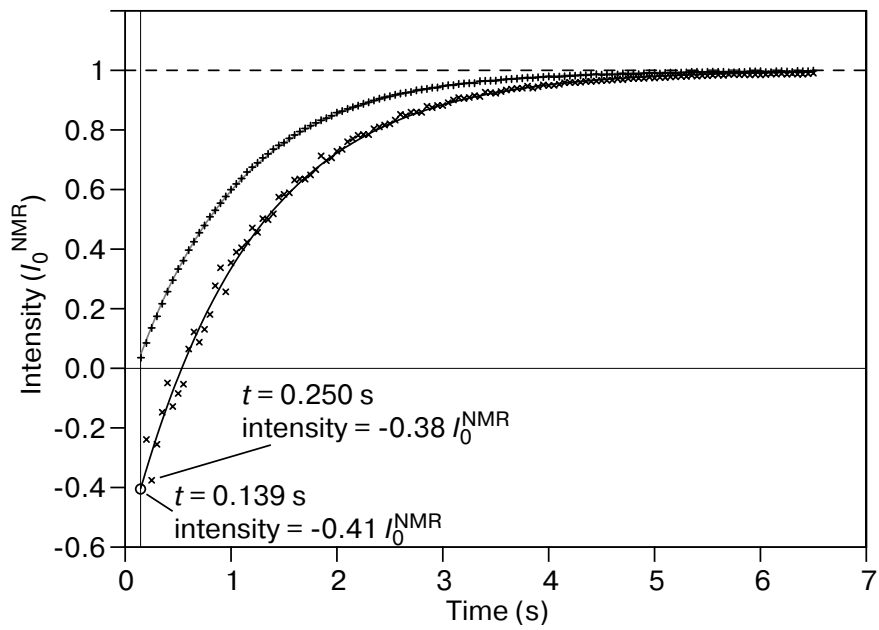

Fig. 5. Integrals of the $\mathrm{H}_{2} \mathrm{O}$ resonance in a $5 \mathrm{mM}$ TEMPOL $\mathrm{H}_{2} \mathrm{O}(80 \%) / \mathrm{D}_{2} \mathrm{O}(20 \%)$ solution after shuttling and waiting for the given time. The plotted integrals are normalized to the fitted Boltzmann value (dashed line) at the NMR field. All data points have been acquired following the same pulse and timing protocols using the sample shuttling. The only difference was that the upper data points $(+)$ have been acquired without DNP and the lower data points $(\times)$ have been acquired with DNP. The time axis is the time after the shuttle-down trigger signal. The fitted (solid line) and extrapolated integrals (O) are only valid for times after the sample arrival at $t=139 \mathrm{~ms}$.

Considering the maximum theoretical NMR and EPR enhancement factors of -7.92 and -328.11 , respectively, and using the extrapolated enhancement factor just at the sample arrival, the effective EPR enhancement factor becomes $\varepsilon_{\mathrm{eff}}^{\mathrm{EPR}}=\varepsilon_{\mathrm{max}}^{\mathrm{EPR}} \varepsilon^{\mathrm{NMR}}(t=139 \mathrm{~ms}) / \varepsilon_{\max }^{\mathrm{NMR}} \approx-17$ in the EPR magnet, assuming that no magnetization was lost during the sample transfer. As the measured initial EPR enhancement factor was -27 before the sample transfer, the value of -17 calculated above indicates that about $40 \%$ of the magnetization was indeed lost during the sample transfer.

For a better understanding of the achievable final enhancement, the effect of relaxation during the shuttling time was simulated with the help of the fielddependent $T_{1}$ times measured for water in the presence of $5 \mathrm{mM}$ TEMPOL [13] and of the path-time diagram shown in Fig. 3. If the sample with the magnetization $I^{\mathrm{EPR}}(t=0)=I_{0}^{\mathrm{EPR}} \varepsilon^{\mathrm{EPR}}=I_{0}^{\mathrm{EPR}}(-27)$ in the EPR magnet could be transferred instantaneously to the NMR magnet, this would correspond to an enhancement factor of $\varepsilon^{\mathrm{NMR}}=\varepsilon^{\mathrm{EPR}} B^{\mathrm{EPR}} / B^{\mathrm{NMR}}=-0.65$. Our simulations indicate a reduction from $\varepsilon^{\mathrm{NMR}}=-0.65$ to -0.50 after a $115 \mathrm{~ms}$ shuttling event, following the path-time diagram in Fig. 3. As the initial DNP signal is recorded $250 \mathrm{~ms}$ after the shuttling was initiated, including $24 \mathrm{~ms}$ dead time and $115 \mathrm{~ms}$ sample transfer, and as further the relaxation occurs at $14.09 \mathrm{~T}$ at a measured rate of $0.875 \mathrm{~s}^{-1}$ for $111 \mathrm{~ms}$, the enhancement factor is predicted to drop further to -0.36 . The experimentally observed enhancement factor of -0.38 is in good agreement with this simulation result (Fig. 6). 


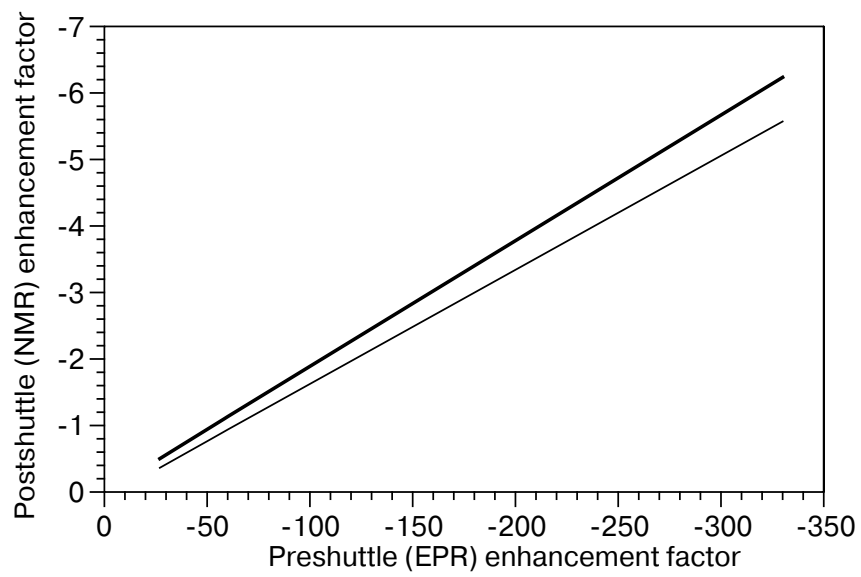

Fig. 6. Thick line indicates the predicted achievable postshuttle enhancement factor $\varepsilon^{\mathrm{NMR}}$ for water in a $5 \mathrm{mM}{ }^{14} \mathrm{~N}$-TEMPOL solution as a function of the preshuttle enhancement factor $\varepsilon^{\mathrm{EPR}}$. It is the result of simulating the relaxation loss of magnetization by shuttling from 0.34 to $14.09 \mathrm{~T}$ for a shuttle time of $115 \mathrm{~ms}$ according to the path-time and relaxation rate measurements (Fig. 5). Thin line indicates $\varepsilon^{\mathrm{NMR}}$ considering shuttling and an additional waiting time of $111 \mathrm{~ms}$ at $14.09 \mathrm{~T}$ to reach stable mesurement conditions.

Using a $5 \mathrm{mM}{ }^{15} \mathrm{~N}-16 \mathrm{D}-\mathrm{TEMPONE}$ solution, a preshuttle enhancement factor of -100 has been reached [12]. Our simulations in Fig. 6 predict that by eliminating the $111 \mathrm{~ms}$ stabilization period and achieving the preshuttle enhancement factor of -100 , the final NMR enhancement factor $\varepsilon^{\mathrm{NMR}}$ would be -1.92 .

\section{Conclusions}

We built a shuttle DNP spectrometer operating at $9.7 \mathrm{GHz}$ with a commercial $0.34 \mathrm{~T}$ EPR magnet mounted on the top of the $14.09 \mathrm{~T}$ cryomagnet of a $600 \mathrm{MHz}$ NMR spectrometer having a distance of $1.525 \mathrm{~m}$ between the EPR cavity and the NMR detection coil. The low field of $0.34 \mathrm{~T}$ was chosen for DNP to maximize the volume of the sample and to minimize the heating effects during the EPR saturation period. Experiments were performed on a $5 \mathrm{mM}$ TEMPOL solution in $\mathrm{H}_{2} \mathrm{O} / \mathrm{D}_{2} \mathrm{O}$. The enhancement reached in the EPR magnet was measured to be -27 . On the basis of our estimates on the coupling and saturation factors for TEMPOL [14] and previous comparative data on TEMPOL and TEMPO [15], the enhancement factor of -27 is presumably one third of the maximum achievable with a $5 \mathrm{mM}$ TEMPOL solution. This discrepancy is not yet understood but it opens the perspective that higher enhancements should be achievable with further optimization of the setup.

Shuttling was achieved in $115 \mathrm{~ms}$, while additional $111 \mathrm{~ms}$ were required to achieve a stable NMR signal. Due to relaxation during these periods, the enhancement factor at the NMR field deteriorated from -0.41 to -0.38 . Clearly, the shuttle process should be accelerated to minimize relaxation losses during the 
travel time and the sample should arrive at the high-field magnet with almost no kinetic energy to be able to record the signal directly after the sample has reached the high-field NMR spectrometer. Using a $5 \mathrm{mM}{ }^{15} \mathrm{~N}-16 \mathrm{D}-\mathrm{TEMPONE}$ solution, by eliminating the stabilization period and utilizing the previously achieved preshuttle enhancement factor of -100 [12], the final NMR enhancement factor $\varepsilon^{\mathrm{NMR}}$ would become -1.92 . Further improvements are possible by reducing relaxational losses during the sample transfer by increasing the magnetic field on the transfer track either using a dual center magnet or an auxiliary transfer field.

Note added in proof. In the meantime we have achieved enhancement of a factor of -3.5 by further optimizing the setup as anticipated in the paper.

\section{Acknowledgments}

We kindly acknowledge the design and constructional contributions of Christian Klaba, Bernd Henkner, Michael Zigan, Mario Lengauer and Rainer Schürkötter from the precision-engineering workshop of the MPIBPC, as well as the technical support of Herbert Nolte from the glass techniques workshop of the same institute. This work was funded by the BioDNP EU Initiative, the Max Planck Society (M.B., C.G., T.C.) and the Fonds der Chemischen Industrie.

\section{References}

1. Ardenkjaer-Larsen, J.H., Fridlund, B., Gram, A., Hansson, G., Hansson, L., Lerche, M.H., Servin, R., Thaning, M., Golman, K.: Proc. Natl. Acad. Sci. USA 100, 10158-10163 (2003)

2. Golman, K., Ardenkjaer-Larsen, J.H., Petersson, J.S., Mansson, S., Leunbach, I.: Proc. Natl. Acad. Sci. USA 100, 10435-10439 (2003)

3. Joo, C-G., Hu, K.-N., Bryant, J. A., Griffin, R.G.: J. Am. Chem. Soc. 128, $9428-9432$ (2006)

4. Purcell, E.M., Pound, R.V.: Phys. Rev. 81, 279-280 (1951)

5. Noack, F.: Prog. Nucl. Magn. Reson. Spectrosc. 18, 171-276 (1986)

6. Redfield, A.G.: Magn. Reson. Chem. 41, 753-768 (2003)

7. Victor, K., Kavolius, V., Bryant, R.G.: J. Magn. Reson. 171, 253-257 (2004)

8. Grosse, S., Gubaydullin, F., Scheelken, H., Vieth, H.M., Yurkovskaya, A.V.: Appl. Magn. Reson. 17, 211-225 (1999)

9. Hausser, K.H., Stehlik, D.: Adv. Magn. Reson. 3, 79-139 (1968)

10. National Institute of Standards and Technology, CODATA recommended values 2006

11. Armstrong, B.D., Han, S.: J. Chem. Phys. 127, 104508 (2007)

12. Höfer, P., Carl, P., Guthausen, G., Reese, M., Carlomagno, T., Griesinger, C., Bennati, M.: Appl. Magn. Reson. 34, 393-398 (2008)

13. Luchinat, C., Parigi, G.: Appl. Magn. Reson. 34, 379-392 (2008)

14. Höfer, P., Parigi, G., Luchinat, C., Carl, P., Guthausen, G., Reese, M., Carlomagno, T., Griesinger, C., Bennati, M.: J. Am. Chem. Soc. 130, 3254-3255 (2008)

15. Gruker, D., Guiberteau, T., Eclancher, B., Chambron, J., Chiarelli, R., Rassat, A., Subra, G., Gallez, B.: J. Magn. Reson. 106 B, 101-109 (1995)

Authors' address: Christian Griesinger, NMR-based Structural Biology, Max Planck Institute for Biophysical Chemistry, 37077 Göttingen, Germany

E-mail: cigr@nmr.mpibpc.mpg.de 